\title{
QUANTUM GROUPS, COHERENT STATES, SQUEEZING AND LATTICE QUANTUM MECHANICS
}

\author{
E.Celeghini ${ }^{1}$, S.De Martino ${ }^{2}$, S.De Siena ${ }^{2}$, M.Rasetti ${ }^{3}$ and G.Vitiello ${ }^{2}$. \\ ${ }^{1}$ Dipartimento di Fisica - Università di Firenze and INFN-Firenze, I 50125 \\ Firenze, Italy \\ ${ }^{2}$ Dipartimento di Fisica - Università di Salerno and INFN-Napoli, I 84100 \\ Salerno, Italy \\ ${ }^{3}$ Dipartimento di Fisica and Unità INFM - Politecnico di Torino, I 10129 \\ Torino, Italy
}

\begin{abstract}
By resorting to the Fock-Bargmann representation, we incorporate the quantum Weyl-Heisenberg algebra, $q$-WH, into the theory of entire analytic functions. The $q-\mathrm{WH}$ algebra operators are realized in terms of finite difference operators in the $z$ plane. In order to exhibit the relevance of our study, several applications to different cases of physical interest are discussed: squeezed states and the relation between coherent states and theta functions on one side, lattice quantum mechanics and Bloch functions on the other, are shown to find a deeper mathematical understanding in terms of $q$-WH. The rôle played by the finite difference operators and the relevance of the lattice structure in the completeness of the coherent states system suggest that the quantization of the $\mathrm{WH}$ algebra is an essential tool in the physics of discretized (periodic) systems.
\end{abstract}

PACS 02.20.+b; 02.90.+p; 03.65.Fd

Annals of Physics (N.Y.), in press. 


\section{Introduction}

A great deal of attention and efforts have been recently devoted in theoretical physics to the mathematical structures referred to as $q$-groups ${ }^{[1],[2],[3]}$.

The basic features of these structures appear now pretty well understood, and promise to be very rich of physical meaning, even though some properties still deserve more study to be fully under control. The interest in $q$-groups arose almost simultaneously in statistical mechanics as well as in conformal theories, in solid state physics as in the study of topologically non-trivial solutions to nonlinear equations, so that as a matter of fact, the research in $q$-groups grew along parallel lines from physical as well as from mathematical problems.

The dual structures to $q$-groups, are the so called $q$-algebras: actually by a now almost universally adopted convention, the name $q$-groups designates $q$-algebras as well, which are instances of Hopf algebras ${ }^{[4]}$.

$q$-algebras are deformations in the enveloping algebras of Lie algebras for which, contrary to the latter, the relevant Hopf algebra features are highly nontrivial, whose structure appears to be an essential tool for the description of composed systems. The general properties of $q$-algebras are better known than those of $q$-groups, in particular for the specific characteristics which relate them with concrete physical models, due to the complexity arising from the $C^{*}$-algebra properties of $q$-groups.

Among the $q$-algebras, the non-semisimple ones are less easy to handle, as in fact it happens in general with non-semisimple structures. We shall here focus our attention on the $q$-deformation of the Weyl-Heisenberg algebra. The WH algebra, admits two inequivalent deformations: one which is properly a $q$-algebra ${ }^{[5]}$, the other (on which we shall focus here our attention, denoting it as $q$-WH, often referred to in the mathematical literature as $\left.\operatorname{osp}_{q}(2 \mid 1)\right)$, originated by the seminal work of Biedenharn ${ }^{[6]}$ and MacFarlane ${ }^{[7]}$, which is characterized by the property that the intrinsic nature of superalgebra - proper also to the WH algebra itself plays a non-trivial role, in view of the form of the coproduct. It can therefore be referred to as a Hopf superalgebra ${ }^{[8],[9]}$.

The $q$-WH algebra has had - in analogy with the non-deformed case - several applications in physics, such as the "quantum" harmonic oscillator, $q$-coherent and squeezed states, Jordan-Wigner realizations, and so on.

In this paper we cast the study of the $q$-WH algebra in the frame of the FockBargmann representation (FBR) of Quantum Mechanics (QM). We show that the analytic structure of the Lie algebra is fully preserved, and we can therefore operate in a scheme where analyticity is ensured. 
As a first step we present a realization of the $q$-WH algebra in terms of finite difference operators. As a result of this we are led to recognize that whenever a finite scale is involved in a self-contained physical theory, a $q$-deformation of the algebra of dynamical observables occurs, with the $q$-parameter related with the finite spacing, namely carrying the information about discreteness. $q$-deformation is also expected in the presence of periodic conditions, since periodicity is but a special form of invariance under finite difference operators.

The analytic properties of the FBR together with the von Neumann lattice topological structure in the complex plane, make then the relation between the $q$-WH algebra and the coherent states (CS) in QM manifest. Here, we obtain a formal relation between the coherent state generator and the commutator of $q$-WH creation and annihilation operators, which is thus recognized to be an operator in the CS space. Moreover, theta functions, in terms of which CS are expressed, also admit a representation in terms of $q$-deformed WH commutators on the von Neumann lattice, allowing us to get further insight in the basic unity of the various structures.

One additional successive step in the understanding of the physical meaning of $q$-deformations is then achieved by realizing that the commutator of $q$-WH creation and annihilation operators acts, in the FBR, like the squeezing generator for CS, a result which confirms a conjecture, previously ${ }^{[10]}$ formulated, whereby $q$-groups are the natural candidates to study squeezed coherent states.

Establishing a relation between CS and the $q$-WH algebra is of course of great interest in view of the numerous interesting physical applications of the CS formalism, and it may also open rich perspectives in Quantum Field Theory where the CS formalism is the key to study vacuum structure and boson condensation.

The relevance of $q$-deformation to discretized system physics naturally leads us to analyze the structure of Lattice Quantum Mechanics (LQM). We study it in configuration space as well as in momentum space and show that LQM is characterized in both cases by the algebra $E(2)$. We complete the analysis of LQM by presenting the lattice CS, optimizing the lattice position-momentum uncertainty relation. $q$-WH turns out to be the algebraic structure underlying the physics of lattice quantum systems. We find that the commutator between $q$-WH creation and annihilation operators acts as generator of the $U(1)$ subgroup of $E(2)$, giving rise to phase variations in the complex plane. In this context, in the presence of a periodic potential on the lattice, there emerges naturally a relation between the $q$-WH algebra and the Bloch functions, which further confirms the conjectured deeply rooted presence of $q$-deformation within the dynamical structure of periodic systems. 
As a general remark, we would like to stress that it is only by fully exploiting the FBR that we succeed in incorporating $q$-deformation of the WH algebra into the theory of (entire) analytical functions. Such result may deserve by itself further attention: in this way, indeed, it appears possible to elucidate the deep rôle of $q$-WH algebra in the physics of lattice quantum systems, coherent states and squeezing.

One result of our analysis which also is remarkable and which will be widely used in the following sections is the one which shows that the action of linear operators of $q-\mathrm{WH}$ algebra may be represented in the FBR as the action of nonlinear operators realized in FBR, e.g. the CS displacement operator.

In this paper we do not pursue to its full extent the study of the rôle played by the complete Hopf (super-)algebra structure of $q-\mathrm{WH}$, that we expect to enter into play when the analysis described here is extended to more degrees of freedom. In particular, we believe the notion of co-product will provide the way of encompassing in a unique structure the non-trivial topological features proper to the extension to higher dimension of theta functions, and hence to several degrees of freedom of coherent states. Such an analysis needs further and deeper formal investigation, which goes beyond the task of present paper (concerned mainly with the possibility of relating the $q$-deformation parameter with some physical quantity) and which we plan for future work.

Through this paper we shall use units such that all relevant physical quantities are dimensionless.

\section{2. $q$-Weyl-Heisenberg algebra, Fock-Bargmann representation and fi- nite difference operators}

The Weyl-Heisenberg algebra is generated by the set of operators $\left\{a, a^{\dagger}, \mathbb{I}\right\}$ with commutation relations

$$
\left[a, a^{\dagger}\right]=\mathbb{I} \quad, \quad[N, a]=-a \quad, \quad\left[N, a^{\dagger}\right]=a^{\dagger}
$$

where $N \equiv a^{\dagger} a$. The Fock space of states $\mathcal{K}$ is nothing but the representation of (2.1) generated by the eigenkets of $N$ with integer (positive and zero) eigenvalues. Any state vector $\mid \psi>$ in $\mathcal{K}$ is thus described by the set $\left\{c_{n} \mid c_{n} \in \mathbb{C}\right\}$ defined by $\left|\psi>=\sum_{n=0}^{\infty} c_{n}\right| n>$, i.e. by its expansion on the complete orthonormal set of eigenkets $\{\mid n>\}$ of $N$.

The intrinsic nature of superalgebra of such scheme is manifestly shown by noticing that, upon defining $H \equiv N+\frac{1}{2}$, the three operators $\left\{a, a^{\dagger}, H\right\}$ close, on 
$\mathcal{K}$, the relations

$$
\left\{a, a^{\dagger}\right\}=2 H \quad, \quad[H, a]=-a \quad, \quad\left[H, a^{\dagger}\right]=a^{\dagger} \quad,
$$

that are, once more on $\mathcal{K}$, equivalent to (2.1).

We shall show in the sequel that the physical interpretation of quantum algebraic structures in the frame of discrete systems stems out, quite naturally, from the quantization of the latter form, which - contrary to the quantization of (2.1) of ref. [5], which preserves the nature of algebra - preserves the nature of superalgebra.

The quantum (deformed) version of $(2.2), q$-WH, is, in terms of the set of operators $\left\{a_{q}, \bar{a}_{q}, H ; q \in \mathbb{C}\right\}$ (where $H$ is assumed to be the same as in $\left.(2.2)\right)^{[8][9]}$ :

$$
\left\{a_{q}, \bar{a}_{q}\right\}=[2 H]_{\sqrt{q}} \quad, \quad\left[H, \bar{a}_{q}\right]=\bar{a}_{q} \quad, \quad\left[H, a_{q}\right]=-a_{q} \quad,
$$

where we utilized the customary notation

$$
[x]_{q} \equiv \frac{q^{\frac{1}{2} x}-q^{-\frac{1}{2} x}}{q^{\frac{1}{2}}-q^{-\frac{1}{2}}} .
$$

The $q$-WH structure defined by (2.3) together with the related coproduct

$$
\begin{gathered}
\Delta(H)=H \otimes \mathbb{I}+\mathbb{I} \otimes H \quad \Rightarrow \quad \Delta(N)=N \otimes \mathbb{I}+\mathbb{I} \otimes N+\frac{1}{2} \mathbb{I} \otimes \mathbb{I} \\
\Delta\left(a_{q}\right)=a_{q} \otimes q^{\frac{1}{4} H}+q^{-\frac{1}{4} H} \otimes a_{q} \quad, \quad \Delta\left(\bar{a}_{q}\right)=\bar{a}_{q} \otimes q^{\frac{1}{4} H}+q^{-\frac{1}{4} H} \otimes \bar{a}_{q}
\end{gathered}
$$

is a quantum superalgebra (graded Hopf algebra) and, consequently, all relations (2.3) are preserved under the coproduct map.

In the space $\mathcal{K}$ (i.e. in the space spanned by the vectors $\{|n>| n \in \mathbb{N}\}$ ), eqs. (2.3) can be rewritten in a more familiar equivalent form ${ }^{[6],[7],[9]}$, which makes them more explicitly analogous to the undeformed case:

$$
a_{q} \bar{a}_{q}-q^{-\frac{1}{2}} \bar{a}_{q} a_{q}=q^{\frac{1}{2} N}, \quad\left[N, a_{q}\right]=-a_{q} \quad, \quad\left[N, \bar{a}_{q}\right]=\bar{a}_{q} ;
$$

or, by introducing $\hat{a}_{q} \equiv \bar{a}_{q} q^{N / 2}$,

$$
\left[a_{q}, \hat{a}_{q}\right] \equiv a_{q} \hat{a}_{q}-\hat{a}_{q} a_{q}=q^{N}, \quad\left[N, a_{q}\right]=-a_{q} \quad, \quad\left[N, \hat{a}_{q}\right]=\hat{a}_{q} \quad .
$$

It must be stressed that (2.1) is a true Hopf algebra, whereas (2.5) and (2.6) are only deformations at the algebra level of (2.1). Thus (2.3-4) is the relevant mathematical structure of our problem. We prefer however to resort henceforth to (2.6) - even though the whole discussion which will follow could, in principle, 
be based on $(2.3)$ - since it is perfectly correct as far as we remain in $\mathcal{K}$ (and in its representations) and it is the most similar to the usual form (2.1) of the WH algebra.

$q$ will be assumed to be any complex number, except in Sect. 5, where it will be restricted to be unimodular. The notion of hermiticity for the generators of $q$-WH associated with complex $q$ is non trivial and has been studied in ref. [10] in connection with the discussion of the feature of squeezing of the generalized coherent states $(G C S)_{q}$ over $\mathcal{K}^{[10]}$.

The basic point of this paper is the functional realization of eqs. (2.6) by means of finite difference operators in the complex plane, in the Fock-Bargmann representation (FBR) of Quantum Mechanics ${ }^{[11],[12]}$. In the FBR state vectors are described by entire analytic functions, contrary to the usual coordinate or momentum representation, where no condition of analyticity is imposed.

The Fock-Bargmann representation of the commutation relations (2.1) is:

$$
N \rightarrow z \frac{d}{d z} \quad, \quad a^{\dagger} \rightarrow z \quad, \quad a \rightarrow \frac{d}{d z}
$$

The corresponding eigenkets of $N$ (orthonormal under the usual gaussian measure $\left.d \mu(z)=\frac{1}{\pi} e^{-|z|^{2}} d z d \bar{z}\right)$ are

$$
u_{n}(z)=\frac{z^{n}}{\sqrt{n !}} \quad, \quad u_{0}(z)=1 \quad\left(n \in \mathbb{N}_{+}\right)
$$

The FBR is the Hilbert space generated by the $u_{n}(z)$, i.e. the whole space $\mathcal{F}$ of entire analytic functions. To each state vector $|\psi\rangle$ is associated, in a one-to-one way, a function $\psi(z) \in \mathcal{F}$ by:

$$
\left|\psi>=\sum_{n=0}^{\infty} c_{n}\right| n>\quad \rightarrow \quad \psi(z)=\sum_{n=0}^{\infty} c_{n} u_{n}(z)
$$

Note also that, as expected in view of the correspondence $\mathcal{K} \rightarrow \mathcal{F}$ (induced by $\left.\mid n>\rightarrow u_{n}(z)\right)$,

$$
\begin{gathered}
a^{\dagger} u_{n}(z)=\sqrt{n+1} u_{n+1}(z), \quad a u_{n}(z)=\sqrt{n} u_{n-1}(z), \\
N u_{n}(z)=a^{\dagger} a u_{n}(z)=z \frac{d}{d z} u_{n}(z)=n u_{n}(z) .
\end{gathered}
$$

(2.10) establish the mutual conjugation of $a$ and $a^{\dagger}$ in the FBR, with respect to the measure $d \mu(z)$. 
Let us now consider the finite difference operator $\mathcal{D}_{q}$ defined by:

$$
\mathcal{D}_{q} f(z)=\frac{f(q z)-f(z)}{(q-1) z}
$$

with $f(z) \in \mathcal{F}, q=e^{\zeta}, \zeta \in \mathbb{C} . \mathcal{D}_{q}$ is the so called $q$-derivative operator ${ }^{[13]}$, which, for $q \rightarrow 1$ ( $\zeta \rightarrow 0$ ), reduces to the standard derivative. By using (2.8) and (2.10), it may be written on $\mathcal{F}$ as

$$
\mathcal{D}_{q}=((q-1) z)^{-1}\left(q^{z \frac{d}{d z}}-1\right)=q^{\frac{z}{2} \frac{d}{d z}} \frac{1}{z}\left[z \frac{d}{d z}\right]_{q}
$$

Consistency between (2.12) and the latter form of $\mathcal{D}_{q}$ can be proven by first "normal ordering" the operator $\left(z \frac{d}{d z}\right)^{n}$ in the form:

$$
\left(z \frac{d}{d z}\right)^{n}=\sum_{m=1}^{n} \mathcal{S}_{n}^{(m)} z^{m} \frac{d^{m}}{d z^{m}}
$$

where $\mathcal{S}_{n}^{(m)}$ denotes the Stirling numbers of the second kind, defined by the recursion relations ${ }^{[14]}$

$$
\mathcal{S}_{n+1}^{(m)}=m \mathcal{S}_{n}^{(m)}+\mathcal{S}_{n}^{(m-1)}
$$

and then expanding in formal power series the exponential $\left(q^{z \frac{d}{d z}}-1\right)$, keeping in mind the identity:

$$
\frac{1}{m !}\left(e^{\theta}-1\right)^{m}=\sum_{n=m}^{\infty} \mathcal{S}_{n}^{(m)} \frac{\theta^{n}}{n !}
$$

$\mathcal{D}_{q}$ satisfies, together with $z$ and $z \frac{d}{d z}$, the commutation relations:

$$
\left[\mathcal{D}_{q}, z\right]=q^{z \frac{d}{d z}} \quad, \quad\left[z \frac{d}{d z}, \mathcal{D}_{q}\right]=-\mathcal{D}_{q} \quad, \quad\left[z \frac{d}{d z}, z\right]=z
$$

which can be recognized as a realization of relations (2.6) in the space $\mathcal{F}$, with the identification

$$
N \rightarrow z \frac{d}{d z} \quad, \quad \hat{a}_{q} \rightarrow z \quad, \quad a_{q} \rightarrow \mathcal{D}_{q} \quad
$$

with $\hat{a}_{q}=\hat{a}_{q=1}=a^{\dagger}$ and $\lim _{q \rightarrow 1} a_{q}=a$ on $\mathcal{F}$. Let us stress that, while $(2.17)$ are restricted to $\mathcal{F}$, the operators $(2.18)$ are related to the true algebraic structure (2.3-4). This must be carefully kept in mind when considering their action over $\mathcal{F} \otimes \mathcal{F}$. 
The relations analogous to (2.10) for the quantum case are

$$
\hat{a}_{q} u_{n}(z)=\sqrt{n+1} u_{n+1}(z) \quad, \quad a_{q} u_{n}(z)=q^{\frac{n-1}{2}} \frac{[n]_{q}}{\sqrt{n}} u_{n-1}(z)
$$

There follows that the $q$-commutator $\left[a_{q}, \hat{a}_{q}\right]$ is defined on the whole $\mathcal{F}$, where it acts as

$$
\left[a_{q}, \hat{a}_{q}\right] f(z)=q^{N} f(z)=f(q z)
$$

Because quantization of the algebra has been essentially obtained by replacing the customary derivative with the finite difference operator, the above discussion suggests ${ }^{[15]}$ that whenever one deals with some finite scale (e.g. with some discrete structure, lattice or periodic system) which cannot be reduced to the continuum by a limiting procedure, then a deformation of the operator algebra acting in $\mathcal{F}$ should arise. Deformation of the operator algebra is also expected whenever the system under study involves periodic (analytic) functions, since periodicity is but a special invariance under finite difference operators.

We finally note that eq. (2.20) provides a remarkable result since it shows that the action of the $q$-WH algebra commutator $\left[a_{q}, \hat{a}_{q}\right]$, which is a linear form in $a_{q}$ and $\hat{a}_{q}$, may be represented in the FBR as the action of the operator $q^{N}$ which is nonlinear in the FBR operators $a$ and $a^{\dagger}$.

In the sections to follow, we shall apply such a result as well as the concepts discussed above to several different cases of physical interest.

\section{3. $q$-Weyl-Heisenberg algebra and coherent states}

In this section we shall discuss the connection between the $q$-WH algebra and the CS formalism, exploiting the theory of entire analytic functions. More specifically, as an application of the result expressed by eq.(2.20), we shall show that the action of the commutator $\left[a_{q}, \hat{a}_{q}\right]$ may be related in the FBR to the action of the CS displacement operator.

The Fock-Bargmann representation provides a simple and transparent frame to describe the usual $\mathrm{CS}^{[12],[16]}$, which are written in the form:

$$
\begin{gathered}
|\alpha>=\mathcal{D}(\alpha)| 0>\quad ; \quad a|\alpha>=\alpha| \alpha>\quad, \quad a \mid 0>=0 \quad, \quad \alpha \in \mathbb{C} \quad, \\
\left|\alpha>=\exp \left(\frac{-|\alpha|^{2}}{2}\right) \sum_{n=0}^{\infty} \frac{\alpha^{n}}{\sqrt{n !}}\right| n>=\exp \left(\frac{-|\alpha|^{2}}{2}\right) \sum_{n=0}^{\infty} u_{n}(\alpha) \mid n>\quad .
\end{gathered}
$$


The relation between the CS and the entire analytic function basis $\left\{u_{n}(z)\right\}$ (eq. (2.8)) is here made explicit: $u_{n}(\alpha)=\mathrm{e}^{\frac{1}{2}|\alpha|^{2}}<n \mid \alpha>$. The unitary displacement operator $\mathcal{D}(\alpha)$ in (3.1) is given by:

$$
\mathcal{D}(\alpha)=\exp \left(\alpha a^{\dagger}-\bar{\alpha} a\right)=\exp \left(-\frac{|\alpha|^{2}}{2}\right) \exp \left(\alpha a^{\dagger}\right) \exp (-\bar{\alpha} a),
$$

for which the following relations hold

$$
\begin{gathered}
\mathcal{D}(\alpha) \mathcal{D}(\beta)=\exp (i \operatorname{Im}(\alpha \bar{\beta})) \mathcal{D}(\alpha+\beta), \\
\mathcal{D}(\alpha) \mathcal{D}(\beta)=\exp (2 i \operatorname{Im}(\alpha \bar{\beta})) \mathcal{D}(\beta) \mathcal{D}(\alpha) .
\end{gathered}
$$

Eq. (3.4) is nothing but the WH group law, also referred to as the Weyl integral representation.

It is well known that, in order to extract a complete set of $\operatorname{CS}\left\{\mid \alpha_{\mathbf{n}}>\right\}$, from the overcomplete set $\{\mid \alpha>\}$ it is necessary to introduce a regular lattice $L$ in the $\alpha$-complex plane ${ }^{[11]}$. The points (lattice vectors) $\alpha_{\mathbf{n}}$ of $L \quad\left(\left\{\alpha_{\mathbf{n}} \in \mathbb{C} ; \mathbf{n}=\right.\right.$ $\left.\left.\left(n_{1}, n_{2}\right) ; n_{j} \in \mathbb{Z}\right\}\right)$ are given by $\alpha_{\mathbf{n}}=n_{1} \Omega_{1}+n_{2} \Omega_{2} \equiv \mathbf{n} \cdot \boldsymbol{\Omega}$, with the two lattice periods $\Omega_{j}, j=1,2$ linearly independent, i.e. such that $\operatorname{Im} \Omega_{1} \bar{\Omega}_{2} \neq 0$.

By closely following ref. [12], we recall that the set $\left\{\mid \alpha_{\mathbf{n}}>\right\}$ (with exclusion of the vacuum state $\left.|0>\equiv| \alpha_{\mathbf{0}}>\right)$ can be shown to be complete, invoking square integrability along with analyticity ${ }^{[17]}$, if the lattice elementary cell has area $\operatorname{Im} \Omega_{1} \bar{\Omega}_{2}=\pi$ ( $L$ is called, in this case, the von Neumann lattice).

The lattice vectors $\alpha_{\mathbf{n}}$ describe the discrete translation invariance of $L$ :

$$
\alpha_{\mathbf{n}+\mathbf{m}}=\alpha_{\mathbf{n}}+\alpha_{\mathbf{m}}
$$

namely,

$$
\mathrm{e}^{\alpha_{\mathbf{n}} \frac{d}{d \alpha}}|\alpha>|_{\alpha=\alpha_{\mathbf{m}}}=\mid \alpha_{\mathbf{n}+\mathbf{m}}>.
$$

We now map the denumerable set of points $\alpha_{\mathbf{n}}$ onto the set $\left\{z_{\mathbf{n}} \mid z_{\mathbf{n}} \in \mathbb{C}\right\}$ with $z_{\mathbf{n}} \equiv \mathrm{e}^{\alpha_{\mathbf{n}}}$. Assuming that the two periods $\Omega_{1}$ and $\Omega_{2}$ have imaginary parts incommensurate with $\pi$ and among themselves, such a map is one-to-one and no point $z_{\mathbf{n}}$ lies on the real axis in the $z$ plane (notice that the set $\left\{z_{\mathbf{n}}\right\}$ does not constitute a lattice in $z$, but it has the structure of concentric circles of radius $\left.\mathrm{e}^{\operatorname{Re} \alpha_{\mathrm{n}}}\right)$.

On the other hand, the function $z=\mathrm{e}^{\alpha}$, which most naturally interpolates among these points, is analytical in its domain of definition, and one may introduce, along with the basis functions $\left\{u_{n}(\alpha)\right\}$ the new functions $\left\{\tilde{u}_{n}(z) \equiv\right.$ $\left.u_{n}(\ln z)=u_{n}(\alpha)\right\}$, with $\tilde{u}_{n}(z) \in \mathcal{F}$. 
Introducing the complex parameter $q_{\mathbf{m}} \equiv \mathrm{e}^{\alpha_{\mathbf{m}}}$, it is then straightforward to check that, in $\mathcal{F}$,

$$
\left[a_{q_{\mathbf{m}}}, \hat{a}_{q_{\mathbf{m}}}\right] \tilde{u}_{n}(z)=q_{\mathbf{m}}{ }^{z \frac{d}{d z}} \tilde{u}_{n}(z)=\tilde{u}_{n}\left(q_{\mathbf{m}} z\right)=u_{n}\left(\alpha+\alpha_{\mathbf{m}}\right)=q_{\mathbf{m}}^{\frac{d}{d \alpha}} u_{n}(\alpha)
$$

where we used eq. (2.20). We have, therefore,

$$
\begin{aligned}
& {\left.\left[a_{q_{\mathbf{n}}}, \hat{a}_{q_{\mathbf{n}}}\right] \tilde{f}_{m}(z)\right|_{z=z_{\mathbf{r}}}=\tilde{f}_{m}\left(q_{\mathbf{n}} z_{\mathbf{r}}\right)=f_{m}\left(\alpha_{\mathbf{r}}+\alpha_{\mathbf{n}}\right)} \\
& =\left.q_{\mathbf{n}}{ }^{\frac{d}{d \alpha}} f_{m}(\alpha)\right|_{\alpha=\alpha_{\mathbf{r}}}=\exp \left(-i \operatorname{Im} \alpha_{\mathbf{n}} \bar{\alpha}_{\mathbf{r}}\right)<m\left|\mathcal{D}\left(\alpha_{\mathbf{n}}\right)\right| \alpha_{\mathbf{r}}>
\end{aligned}
$$

where we utilized eq. (3.4), together with the notation $f_{m}(\alpha) \equiv \exp \left(-\frac{|\alpha|^{2}}{2}\right) u_{m}(\alpha)$.

The operator $\left.\left[a_{q}, \hat{a}_{q}\right]\right|_{q=q_{\mathbf{n}}}$, which realizes the mapping $\tilde{f}\left(z_{\mathbf{m}}\right) \mapsto \tilde{f}\left(q_{\mathbf{n}} z_{\mathbf{m}}\right)$, can, in such way, be thought of as extendible to the map on the $\alpha$-plane $\mid \alpha_{\mathbf{m}}>\mapsto$ $\mid \alpha_{\mathbf{n}+\mathbf{m}}>$.

We also observe that in the FBR we have, for any $f \in \mathcal{F}$

$$
\mathcal{D}(\beta) f(\alpha)=\exp \left(-\frac{|\beta|^{2}}{2}\right) \exp (\alpha \beta) f(\alpha-\bar{\beta}) \quad, \quad f \in \mathcal{F} \quad,
$$

so that, in view of eq. (3.8), we can write, for $q=\mathrm{e}^{\zeta}$ and, once more, $z=\mathrm{e}^{\alpha}$,

$$
\left[a_{q}, \hat{a}_{q}\right] \tilde{f}(z)=\mathrm{e}^{\frac{1}{2}|\zeta|^{2}} \bar{q}^{\alpha} \mathcal{D}(-\bar{\zeta}) f(\alpha)
$$

Eqs. (3.9) and (3.11) show the relation between the $q-\mathrm{WH}$ algebra operator $\left[a_{q}, \hat{a}_{q}\right]$ and the CS displacement operator, establishing a relation between the quantum algebra (2.6) (or equivalently, at this level, (2.3)), in the frame of the theory of entire analytical functions, and the theory of CS.

Once more we are led to conclude that the existence of a quantum deformed algebra signals the presence of finite lengths in the theory and provides the natural framework for the physics of discretized systems, the $q$-deformation parameter being related with the lattice constants.

The lattice structure is also of crucial relevance in the relation between theta functions and the complete system of CS.

In order to establish such a relation, we look for the common eigenvectors $|\theta\rangle$ of the CS operators $\mathcal{D}\left(\alpha_{\mathbf{n}}\right)$ associated to the regular lattice $L{ }^{[12]}$. A common set of eigenvectors exists if and only if all the $\mathcal{D}\left(\alpha_{\mathbf{n}}\right)$ commute, i.e. when the $\mathcal{D}\left(\Omega_{j}\right)$ commute, as indeed it happens on the von Neumann lattice (cf. eq.(3.5)).

The eigenstates $\mid \theta>$ are characterized by two real numbers $\epsilon_{1}$ and $\epsilon_{2}, \mid \theta>\equiv$ $\mid \theta_{\epsilon}>$, eigenvectors of $\mathcal{D}\left(\Omega_{i}\right)$ :

$$
\mathcal{D}\left(\Omega_{j}\right)\left|\theta_{\epsilon}>=e^{i \pi \epsilon_{j}}\right| \theta_{\epsilon}>\quad, \quad j=1,2 \quad, \quad 0 \leq \epsilon_{j} \leq 2
$$


In other words, $\left|\theta_{\epsilon}\right\rangle$, which belongs to a space which is the extension ${ }^{[12]}$ of the Hilbert space where the operators $\mathcal{D}(\alpha)$ act, corresponds to a point on the twodimensional torus. The action of $\mathcal{D}(\alpha)$ on $\left|\theta_{\epsilon}\right\rangle$ generates a set of generalized coherent states. Use of eqs. (3.12) and (3.4) gives

$$
\mathcal{D}\left(\alpha_{\mathbf{m}}\right)\left|\theta_{\epsilon}>=e^{i \pi F_{\epsilon}(\mathbf{m})}\right| \theta_{\epsilon}>
$$

with $\alpha_{\mathbf{m}}=\mathbf{m} \cdot \boldsymbol{\Omega}$, an arbitrary lattice vector, and

$$
F_{\epsilon}(\mathbf{m})=m_{1} m_{2}+m_{1} \epsilon_{1}+m_{2} \epsilon_{2}
$$

On the other hand, the system of CS is associated, in the FBR, with a corresponding set of entire analytic functions, say $\theta_{\epsilon}(\alpha)$. Eq. (3.10) with $\bar{\alpha}=-\alpha_{\mathbf{m}}$ shows that eq. (3.13) may be written as

$$
\theta_{\epsilon}\left(\alpha+\alpha_{\mathbf{m}}\right)=\exp \left(i \pi F_{\epsilon}(-\mathbf{m})\right) \exp \left(\frac{\left|\alpha_{\mathbf{m}}\right|^{2}}{2}\right) \exp \left(\bar{\alpha}_{\mathbf{m}} \alpha\right) \theta_{\epsilon}(\alpha)
$$

which is the functional equation for the theta functions ${ }^{[12],[18],[19]}$. We emphasize that such relation is obtained by considering the CS system corresponding to the von Neumann lattice $L$. The relation with the $q$-WH algebra is established by realizing that in $\mathcal{F}$ the functional equation (3.15) reads

$$
\left[a_{q_{\mathbf{m}}}, \bar{a}_{q_{\mathbf{m}}}\right] \tilde{\theta}(z)=\tilde{\theta}\left(q_{\mathbf{m}} z\right)=\exp \left(i \pi F_{\epsilon}(-\mathbf{m})\right) \exp \left(\frac{\left|\alpha_{\mathbf{m}}\right|^{2}}{2}\right) \exp \left(\bar{\alpha}_{\mathbf{m}} \alpha\right) \theta_{\epsilon}(\alpha)
$$

It is interesting to observe that the commutator $\left[a_{q}, \hat{a}_{q}\right]$ acts as shift operator on the Von Neumann lattice whereas it acts as $z$-dilatation operator $(z \rightarrow q z)$ in the space of entire analytic functions or, else, as the $U(1)$ generator of phase variations in the $z$-plane, $\arg (z) \rightarrow \arg (z)+\theta$, when $q=\mathrm{e}^{i \theta}$.

It is remarkable that eqs. (3.8), (3.9) and (3.11) represent the action of the $q$-WH algebra commutator $\left[a_{q}, \hat{a}_{q}\right]$, (bi-)linear in $a_{q}$ and $\hat{a}_{q}$, through the action of the CS displacement operator which is nonlinear in the FBR operators $a$ and $a^{\dagger}$. Conversely, the nonlinear operator $\mathcal{D}(\alpha)$ is represented by the linear form $\left[a_{q}, \hat{a}_{q}\right]$ in the $q$-WH algebra.

\section{4. $q$-Weyl-Heisenberg algebra and the squeezing generator}

Over a Hilbert space of states identified with the space $\mathcal{F}$ of entire analytic functions $\psi(z)$, the identity

$$
2 z \frac{d}{d z} \psi(z)=\left\{\frac{1}{2}\left[\left(z+\frac{d}{d z}\right)^{2}-\left(z-\frac{d}{d z}\right)^{2}\right]-1\right\} \psi(z)
$$


holds. In the present section we set $z \equiv x+i y, x$ denoting the position coordinate variable. For convenience, we introduce the operators

$$
\alpha=\frac{1}{\sqrt{2}}\left(z+\frac{d}{d z}\right) \quad, \quad \alpha^{\dagger}=\frac{1}{\sqrt{2}}\left(z-\frac{d}{d z}\right) \quad, \quad\left[\alpha, \alpha^{\dagger}\right]=\mathbb{I} \quad,
$$

namely, in terms of the FBR operators $a$ and $a^{\dagger}$,

$$
z=\frac{1}{\sqrt{2}}\left(\alpha+\alpha^{\dagger}\right) \rightarrow a \quad, \quad \frac{d}{d z}=\frac{1}{\sqrt{2}}\left(\alpha-\alpha^{\dagger}\right) \rightarrow a^{\dagger} .
$$

One should notice that, in $\mathcal{F}, \alpha^{\dagger}$ is indeed the conjugate of $\alpha$, as thoroughly discussed in Ref. [10] (see also [12]). We observe that, in the limit $y \rightarrow 0, \alpha$ and $\alpha^{\dagger}$ turn into the conventional annihilation and creator operators $a$ and $a^{\dagger}$ associated with $x$ and $p_{x}$ in the canonical configuration representation, respectively.

We shall now prove that the operator

$$
\left[a_{q}, \hat{a}_{q}\right]=\frac{1}{\sqrt{q}} \exp \left(\frac{\zeta}{2}\left(\alpha^{2}-\alpha^{\dagger^{2}}\right)\right) \equiv \frac{1}{\sqrt{q}} \hat{\mathcal{S}}(\zeta)
$$

where $q=e^{\zeta}$ (for simplicity, assumed to be real), which reminds the squeezing operator of quantum optics ${ }^{[20]}$, acts indeed in $\mathcal{F}$ as well as a squeezing operator in the configuration representation in the limit $y \rightarrow 0$.

We note first that, as it is well known ${ }^{[20]}$, the right hand side of (4.3) is an $S U(1,1)$ group element. In fact, by defining $K_{-}=\frac{1}{2} \alpha^{2}, K_{+}=\frac{1}{2} \alpha^{\dagger 2}, K_{z}=$ $\frac{1}{2}\left(\alpha^{\dagger} \alpha+\frac{1}{2}\right)$, one easily checks they close the algebra $s u(1,1)$.

We have also (see eq. (2.20)):

$$
\begin{gathered}
{\left[a_{q}, \hat{a}_{q}\right] \psi(z)=\exp \left(\zeta z \frac{d}{d z}\right) \psi(z)=\frac{1}{\sqrt{q}} \exp \left(\frac{\zeta}{2}\left(\alpha^{2}-\alpha^{\dagger^{2}}\right)\right) \psi(z)} \\
=\frac{1}{\sqrt{q}} \hat{\mathcal{S}}(\zeta) \psi(z) \equiv \frac{1}{\sqrt{q}} \psi_{s}(z)
\end{gathered}
$$

with $\psi_{s}(z)$ the squeezed states in FBR, and in the $y \rightarrow 0$ limit (still in $\mathcal{F}$ )

$$
\begin{gathered}
\hat{\mathcal{S}}^{-1}(\zeta) \alpha \hat{\mathcal{S}}(\zeta) \rightarrow \hat{\mathcal{S}}^{-1}(\zeta) a \hat{\mathcal{S}}(\zeta) \\
\hat{\mathcal{S}}^{-1}(\zeta) \alpha^{\dagger} \hat{\mathcal{S}}(\zeta) \rightarrow \hat{\mathcal{S}}^{-1}(\zeta) a^{\dagger} \hat{\mathcal{S}}(\zeta) \\
\hat{\mathcal{S}}^{-1}(\zeta) z \hat{\mathcal{S}}(\zeta)=\frac{1}{q} z \rightarrow \frac{1}{q} x \quad, \quad \hat{\mathcal{S}}^{-1}(\zeta) p_{z} \hat{\mathcal{S}}(\zeta)=q p_{z} \rightarrow q p_{x}
\end{gathered}
$$

where $p_{z} \equiv-i \frac{d}{d z}$. We thus see that in the limit $y \rightarrow 0$

$$
\begin{aligned}
& \int d \mu(z) \bar{\psi}(z) \hat{\mathcal{S}}^{-1}(\zeta) z \hat{\mathcal{S}}(\zeta) \psi(z) \rightarrow \frac{1}{q}<x> \\
& \int d \mu(z) \bar{\psi}(z) \hat{\mathcal{S}}^{-1}(\zeta) p_{z} \hat{\mathcal{S}}(\zeta) \psi(z) \rightarrow q<p_{x}>
\end{aligned}
$$


so that the root mean square deviations $\Delta x$ and $\Delta p$ of position and momentum satisfy

$$
\Delta x \Delta p=\frac{1}{2} \quad, \quad \Delta x=\frac{1}{q} \sqrt{\frac{1}{2}} \quad, \quad \Delta p=q \sqrt{\frac{1}{2}} .
$$

Manifestly the $q$-deformation parameter plays here the rôle of squeezing parameter and the commutator $\left[a_{q}, \hat{a}_{q}\right]$ acts, up to a numerical factor, like the conventional squeezing generator with respect to the operators $\alpha$ and $\alpha^{\dagger}$.

Let us finally observe that, in view of the holomorphy conditions holding for $f(z) \in \mathcal{F}$

$$
\frac{d}{d z} f(z)=\frac{d}{d x} f(z)=-i \frac{d}{d y} f(z)
$$

one finds

$$
z \frac{d}{d z}=z \frac{d}{d x}=x \frac{d}{d x}+i y \frac{d}{d x}=-i x \frac{d}{d y}+i y \frac{d}{d x}=x p_{y}-y p_{x} \equiv L
$$

with $L$ an angular momentum operator. This implies that, for $\zeta=i \theta$, with $\theta$ real, the commutator $\left[a_{q}, \hat{a}_{q}\right]$ acts in $\mathcal{F}$ as the $U(1)$ group element

$$
\left[a_{q}, \hat{a}_{q}\right]=e^{i \theta L}
$$

\section{Lattice Quantum Mechanics and Bloch functions.}

In this section we first recall the structure of Lattice Quantum Mechanics (LQM) and then relate it to $q$-WH. For completeness we also construct lattice CS minimizing the lattice position-momentum uncertainty relation. Finally, we close with an application to Bloch functions.

We limit ourselves to 1-dimensional lattice: the extension to more dimensions requires the accurate use of the coproduct in the quantum-algebraic scheme, and we do not extend our analysis to such a case in the present paper.

A 1-dimensional lattice quantum system is defined on the configurational Hilbert space $\mathcal{G}=l^{2}(\epsilon \mathbb{Z})$, where $\mathbb{Z}$ denotes the set of integers $n$ and $\epsilon$ is the lattice spacing. The (hermitian) position operator, $\hat{x}_{\epsilon}$, is defined by

$$
\left[\hat{x}_{\epsilon} f\right]\left(x_{n}\right)=x_{n} f\left(x_{n}\right)=\epsilon n f\left(x_{n}\right) \quad, \quad f \in \mathcal{G} \quad,
$$

whereas the (hermitian) lattice momentum operator $\hat{p}_{\epsilon}$ is defined by

$$
\left[\hat{p}_{\epsilon} f\right]\left(x_{n}\right)=-i\left[\mathcal{D}_{\epsilon} f\right]\left(x_{n}\right)
$$


where $\mathcal{D}_{\epsilon}$ is the symmetrized, finite difference gradient

$$
\left[\mathcal{D}_{\epsilon} f\right]\left(x_{n}\right)=(2 \epsilon)^{-1}\left[f\left(x_{n+1}\right)-f\left(x_{n-1}\right)\right]
$$

In this section we use the finite difference operator $\mathcal{D}_{\epsilon}$, which is slightly different from $\mathcal{D}_{q}$, to follow the conventional use in the literature on LQM. The relation between the two operators is manifest: $\mathcal{D}_{\epsilon}=\frac{1}{2}\left(\mathcal{D}_{q_{n}}+\mathcal{D}_{q_{n-1}}\right)$, with $q_{n}=1+n^{-1}$.

The dual momentum-space representation of the above operators is simply

$$
\begin{gathered}
{\left[\hat{x}_{\epsilon} f\right](k)=i \frac{d}{d k} f(k),} \\
{\left[\hat{p}_{\epsilon} f\right](k)=\epsilon^{-1} \sin (k \epsilon) f(k),}
\end{gathered}
$$

respectively, where $f(k)$ is the Fourier conjugate of $f\left(x_{n}\right), k$ belonging to the first Brillouin zone (BZ), $|k| \leq \pi / \epsilon$.

Over $\mathcal{G}$ one has the following commutation relation between position and momentum

$$
\left[\left[\hat{x}_{\epsilon}, \hat{p}_{\epsilon}\right] f\right]\left(x_{n}\right)=\frac{i}{2}\left(f\left(x_{n+1}\right)+f\left(x_{n-1}\right)\right) \equiv i\left[\hat{P}_{\epsilon} f\right]\left(x_{n}\right)
$$

The operator $\hat{P}_{\epsilon}$ has the following explicit expression. Consider the set of entire functions $f(x)$ whose restriction to the lattice points $x_{n}$ is the set $\left\{f\left(x_{n}\right)\right\}$ : then $\hat{P}_{\epsilon}$ is given by $\hat{P}_{\epsilon}=\cos (\epsilon \hat{p})$, with $\hat{p}=-i \frac{d}{d x}$, as it follows from

$$
f\left(x_{n \pm 1}\right) \equiv f\left(x_{n} \pm \epsilon\right)=[\exp ( \pm i \epsilon \hat{p}) f]\left(x_{n}\right)
$$

The operators $\hat{P}_{\epsilon}, \hat{p}_{\epsilon}$ and $\hat{x}_{\epsilon}$ generate the algebra $E(2)$ :

$$
\left[\hat{x}_{\epsilon}, \hat{p}_{\epsilon}\right]=i \hat{P}_{\epsilon} \quad, \quad\left[\hat{x}_{\epsilon}, \hat{P}_{\epsilon}\right]=-i \epsilon^{2} \hat{p}_{\epsilon} \quad, \quad\left[\hat{P}_{\epsilon}, \hat{p}_{\epsilon}\right]=0
$$

which contracts to the Weyl-Heisenberg algebra in the limit $\epsilon \rightarrow 0$ : the discrete lattice spacing $\epsilon$ plays thus the rôle of deformation parameter.

Upon introducing, in momentum space, the angle $\phi=k \epsilon,-\pi \leq \phi \leq \pi$, ranging on the whole unit circle, we set

$$
L_{3}=-i \frac{d}{d \phi}=-\frac{i}{\epsilon} \frac{d}{d k} \quad, \quad L_{1}=\cos \phi \quad, \quad L_{2}=\sin \phi
$$

The algebra (5.6) takes then the more customary $E(2)$ form

$$
\left[L_{3}, L_{1}\right]=i L_{2} \quad, \quad\left[L_{3}, L_{2}\right]=-i L_{1} \quad, \quad\left[L_{1}, L_{2}\right]=0
$$


It is interesting to observe that eqs. (5.8) and (5.9) correspond to the ones reported in ref. [21] in the framework of a discussion on the phase-angle commutation relations.

In order to show that the structure underlying LQM is, once more, $q$-WH, we introduce now - as in sect. 3 - a conformal image $\tilde{\mathcal{F}}$ of the configurational Hilbert space $\mathcal{G}$ in the following way. We map the denumerable set of lattice points $x_{n}=\epsilon n$ onto the set of points $z_{n} \equiv \mathrm{e}^{i x_{n}}$ on the unit circle. Assuming that $\eta=\frac{2 \pi}{\epsilon}$ is an irrational number, such a set $\left\{z_{n}\right\}$ is dense on the circle, $z_{n} \neq z_{m}$ for $n \neq m$ and the number of lattice points mapped on each single image of the circle is the maximum integer less than $\eta$. The function $z=\mathrm{e}^{i x}$ (not to be confused with the $z$ variable introduced in sect. 4 ), which most naturally interpolates among these points, is analytical in its domain of definition. One may therefore introduce the function $\tilde{f}(z) \equiv f(-i \log z)=f(x)$, provided the bridging of the cut is realized by the customary analytic continuation of the $\log$ function. We have

$$
i[\hat{p} f](x)=\frac{d}{d x} f(x)=i z \frac{d}{d z} f(-i \log z)=i z \frac{d}{d z} \tilde{f}(z) \quad, \quad z=e^{i x} \quad, \quad \tilde{f} \in \tilde{\mathcal{F}}
$$

Once more, we restrict the space $\mathcal{F}$ to the space of the functions $\left\{f\left(x_{n}\right) \mid f \in\right.$ $\mathcal{F}$ \} over the lattice points $x_{n}$.

Upon noticing that the operators $A^{\dagger}=e^{i x}, A=-i e^{-i x} \frac{d}{d x}, N=-i \frac{d}{d x}=$ $\hat{p}$ provide a realization of WH-algebra, the form of $A^{\dagger}$ suggests that the same realization of FBR may be adopted in $\tilde{\mathcal{F}}\left(\left\{f\left(x_{n}\right)\right\}\right)$ for the LQM as well. Eq. (5.6) now implies

$$
f\left(x_{n+1}\right)=\left[e^{i \epsilon \hat{p}} f\right]\left(x_{n}\right)=q^{N} \tilde{f}\left(z_{n}\right)=\tilde{f}\left(q z_{n}\right)=\tilde{f}\left(z_{n+1}\right)
$$

where we have set $e^{i \epsilon}=q$, and used eq. (2.20).

This makes clear that the algebraic structure of LQM is intimately related with the $q$-WH algebra, the $q$-deformation parameter being determined by the discrete lattice length $\epsilon=-i \log q$.

The same conclusion can be reached by constructing LQM in momentum space. In such a case one has to consider a conformal image $\tilde{\mathcal{H}}$ of the Hilbert space in the momentum representation, by setting $z=e^{i \phi}$, so that (cf. eq.(5.8))

$$
-i \frac{d}{d \phi}=-\frac{i}{\epsilon} \frac{d}{d k}=z \frac{d}{d z}
$$

On the other hand,

$$
L_{3} f(\phi)=-i \frac{d}{d \phi} f(\phi)=z \frac{d}{d z} \tilde{f}(z)=N \tilde{f}(z) \quad, \quad \tilde{f} \in \tilde{\mathcal{H}}
$$


and eq. (2.20) gives:

$$
\left[a_{q}, \hat{a}_{q}\right] \tilde{f}(z)=q^{N} \tilde{f}(z)=\tilde{f}(q z)=f(\phi+\epsilon)=e^{i \epsilon L_{3}} f(\phi)
$$

The $E(2)$ algebra (5.9) is now realized in the momentum space FBR as

$$
\begin{gathered}
{\left[\left[L_{1}, L_{3}\right] \tilde{f}\right](z)=-i\left[L_{2} \tilde{f}\right](z) \quad, \quad\left[\left[L_{2}, L_{3}\right] \tilde{f}\right](z)=i\left[L_{1} \tilde{f}\right](z)} \\
{\left[\left[L_{1}, L_{2}\right] \tilde{f}\right](z)=0}
\end{gathered}
$$

with $\tilde{f} \in \tilde{\mathcal{H}}$, and the identifications (see eq.(5.8))

$$
L_{1}=\frac{z+\bar{z}}{2}, L_{2}=\frac{z-\bar{z}}{2 i}, L_{3}=z \frac{d}{d z}, L_{+}=z, L_{-}=\bar{z} .
$$

In conclusion, in this representation $\left[a_{q}, \hat{a}_{q}\right]$ is nothing but the $e^{i \epsilon L_{3}}$ group element of $E(2)$. We also note that $z^{n}=e^{i n \phi}, n$ integer, is the eigenfunction of $L_{3}$ associated with the integer eigenvalue $n$ of the number operator in the FBR

$$
L_{3} z^{n}=N z^{n}=n z^{n}
$$

We can now construct the CS minimizing the lattice position-momentum uncertainty relation. To begin with, by following standard procedures ${ }^{[21],[23]}$, we get, in the LQM scheme above, the uncertainty inequalities

$$
\begin{aligned}
\Delta^{2}\left(\hat{x}_{\epsilon}\right) \Delta^{2}\left(\hat{p}_{\epsilon}\right) & \geq \frac{1}{4}\langle\cos (k \epsilon)\rangle^{2}, \\
\Delta^{2}\left(\hat{x}_{\epsilon}\right) \Delta^{2}(\cos (k \epsilon)) & \geq \frac{1}{4} \epsilon^{2}\langle\sin (k \epsilon)\rangle^{2},
\end{aligned}
$$

where $\langle\hat{A}\rangle=\int d k \Psi^{*}(k) \hat{A} \Psi(k)$ denotes quantum expectation on the lattice and $\Delta^{2}(\hat{A})=\left\langle\hat{A}^{2}\right\rangle-\langle\hat{A}\rangle^{2}$ the (square) variance. Relations (5.18) are written in momentum space for convenience, and are consequences of eqs. (5.7). The continuum limit $\epsilon \rightarrow 0$ corresponds to opening the circle into a line. In $d$ dimensions the limit $\epsilon \rightarrow 0$, would be an isometric and conformal mapping of the torus on the plane (decompactification) ${ }^{[22]}$. The states minimizing the uncertainty products of eqs.(5.18) must satisfy ${ }^{[21],[23]}$

$$
\left(\hat{x}_{\epsilon}+i \gamma \hat{p}_{\epsilon}\right) \Psi=\lambda \Psi
$$

where $\lambda=\left\langle\hat{x}_{\epsilon}\right\rangle+i \gamma\left\langle\hat{p}_{\epsilon}\right\rangle$, and $\gamma$ is connected with the position and momentum variances $\Delta\left(\hat{x}_{\epsilon}\right), \Delta\left(\hat{p}_{\epsilon}\right)$. Relation (5.19) becomes, in momentum space,

$$
\left[\frac{d}{d(\epsilon k)}+\bar{\gamma} \sin (\epsilon k)\right] \Psi(k)=-i \bar{\lambda} \Psi(k),
$$


where $\bar{\lambda}=\lambda \epsilon^{-1}, \bar{\gamma}=\gamma \epsilon^{-2}$. Its solution is

$$
\Psi(k)=G \exp [\bar{\gamma} \cos (\epsilon k)-i \bar{\lambda} \epsilon k] \quad,
$$

where the normalization constant $G$ is given by $G=2 \pi \epsilon^{-1} I_{0}(2 \bar{\gamma}), I_{0}$ denoting the modified Bessel function of the first kind of order 0 . Notice that, in the continuum limit $\epsilon \rightarrow 0$, the Fourier transform $\tilde{\Psi}(x)$ of (5.21) becomes

$$
\tilde{\Psi}(x)=(\gamma \pi)^{-\frac{1}{4}} \exp \left\{-\left[(2 \gamma)^{-1}(x-\langle\hat{x}\rangle)^{2}+i\langle\hat{p}\rangle(x-\langle\hat{x}\rangle)\right]\right\}
$$

which is just the minimum uncertainty wavefunction given by Schrödinger ${ }^{[24]}$. By setting $z=\langle x\rangle+i \gamma\langle p\rangle$, eq. (5.22) defines the usual coherent states ${ }^{[12]}$. The wave-function (5.21) is the coherent state for a system with discretized position and momentum or, equivalently, endowed with some periodic constraint.

Finally, we close this section by showing, as a further application of eq. (2.20), that Bloch functions provide a representation of $q-\mathrm{WH}$ algebra. The basic observation is that the functions $z=e^{i \phi}$ and $z^{n}$ play also a crucial rôle in the Bloch function theory ${ }^{[25]}$. Bloch theorem indeed states the existence, in the presence of a periodic potential $V\left(x_{n}\right)=V\left(x_{n}+\epsilon\right)$, of solutions of the related Schrödinger equation of the form

$$
\psi\left(x_{n}\right)=e^{ \pm i k x_{n}} v_{k}\left(x_{n}\right)
$$

with $v_{k}\left(x_{n}\right)=v_{k}\left(x_{n}+\epsilon\right) . \psi\left(x_{n}\right)$ is referred to as a Bloch function. Limiting ourselves for simplicity to considering the plus sign in $(5.23), \psi\left(x_{n}\right)$ has the property

$$
\psi\left(x_{n}+\epsilon\right)=e^{i k \epsilon} \psi\left(x_{n}\right)=z \psi\left(x_{n}\right)
$$

We thus see that the choice of the variable $z=e^{i k \epsilon}$ turns out to be natural in the case of periodic potentials:

$$
\psi\left(x_{n}\right)=z^{n} v_{k}\left(x_{n}\right) \quad, \quad \psi\left(x_{n}+\epsilon\right)=z^{n+1} v_{k}\left(x_{n}\right)
$$

Since $z^{n}=\left(z_{n}\right)^{k}$, from eq. (5.11)

$$
q^{N}\left(z_{n}\right)^{k}=\left(q z_{n}\right)^{k}=\left(e^{i \epsilon} e^{i x_{n}}\right)^{k}=e^{i k \epsilon(n+1)}=z^{n+1},
$$

where $q^{N}$ is understood as defined on $\tilde{\mathcal{F}}$. We thus have in $\tilde{\mathcal{F}}$, by using eq. $(2.20)$,

$$
\psi\left(x_{n}+\epsilon\right)=\left[\left[{ }_{q}, \hat{a}_{q}\right]\left(z_{n}\right)^{k} u_{k}\right]\left(x_{n}\right)=\left[\left[{ }_{q}, \hat{a}_{q}\right] \psi\right]\left(x_{n}\right)
$$

In other words, the condition implementing the Bloch function periodicity features is realized by the same operator in the $q$-WH algebra acting, in the FBR, as dilatation. 


\section{Conclusions}

In this paper we have considered the $q$-deformation of the WH algebra in connection with typical problems in QM: discrete and/or periodic systems, coherent states, squeezing, lattice quantum mechanics, resorting essentially to the tool of finite difference operators. The above examples have been proposed as physically relevant representative of systems where $q$-deformation plays a rôle. Such a collection of applications is not only interesting on its own, but also as a laboratory where problems characterized by the common feature of a discrete structure are treated in the framework of (entire) analytic functions theory.

The underlying aim is the "phylosofical" claim that, whenever one deals with a quantum system defined on a countable set of degrees of freedom, then one has to work in the space of the related analytical functions and the structure of the operator algebra is that of a quantum algebra. In this sense, the term deformation does not sound as the most appropriate, since $q$-algebras provide an algorithm of wide physical application, and not an exception for situations deformed with respect to a standard one. Moreover, quantum algebras stucture amounts to much more than deformation, and in particular we expect that - in the case of $q$-WH - both the graded structure and the operation of co-product have to be taken into account. Such a general philosophy appears to find very concrete preliminary support in the results discussed in this paper.

From the point of view of group theory, we used the well known mapping of a $q$-algebra into the universal enveloping algebra of the corresponding Lie structure; to be specific, the mapping of finite difference operators into functions of differential operators, which can be indeed achieved only by operating on $C^{\infty}$ functions. This was the main reason to work with FBR and to introduce the conformal image of both configuration and momentum space in the study of LQM.

An interesting and natural development, namely the extension from QM to Quantum Field Theory by considering the infinite volume limit of the lattice system, leads to the parametrization of the unitarily inequivalent representations of the canonical commutation relations by means of the deformation parameter $q^{[27]}$. Different values of the lattice spacing are thus described by inequivalent representations. In this framework finite temperature and dissipative systems ${ }^{[26]}$ may find an appropriate unified description.

The notion of coproduct, although an essential tool in the discussion of $q$ algebras, has not explicitly entered so far our analysis, in that in this paper we have mainly focused our attention on the links between the $q$-WH algebra and several structures of physical interest in $1-d$ only. We are aware that a deeper 
understanding of such links may be achieved by investigating the rôle played by the whole structure of Hopf algebra of the $q-\mathrm{WH}$, that we expect should be essential in dealing with systems in higher dimension. This will hold in particular when dealing with the generalized definition of theta functions, where the notion of mapping class group (connected with the braiding features of the transformations of the coherent states manifold) comes into play.

Work is under progress along these latter directions.

\section{Acknowledgements}

We gratefully acknowledge useful discussions with O.V.Man'ko, J.Katriel and A.I.Solomon. 


\section{References.}

[1] Drinfeld V.G., Proc. ICM Berkeley, CA; A.M. Gleason, ed,; AMS, Providence, R.I., 1986, page 798.

[2] Jimbo M., Int. J. of Mod. Phys. A4 (1989) 3759.

[3] Manin Yu.I., Quantum groups and Non-Commutative Geometry, Centre de Recherches Mathématiques, Montreal, 1988.

[4] Abe E., Hopf algebras, Cambridge tracts in Math. no. 74, Cambridge Univ. Press, Cambridge, 1980.

[5] E. Celeghini, R. Giachetti, E. Sorace, and M. Tarlini, J. Math. Phys. 31 (1990) 2548; and 32 (1991) 1155.

[6] L.C Biedenharn, J. Phys. 22 (1989) L873

[7] A.J. Macfarlane, J. Phys. 22 (1989) 4581

[8] P.P. Kulish, and N.Yu. Reshetikin, Lett. Math. Phys. 18 (1989) 143.

[9] E.Celeghini, T.D.Palev and M.Tarlini, Mod. Phys. Lett B5 (1991)187

[10] E. Celeghini, M. Rasetti and G. Vitiello, Phys. Rev. Lett. 66 (1991) 2056

[11] V.A. Fock, Z. Phys. 49 (1928) 339

V. Bargmann, Comm. Pure and Appl. Math. 14 (1961) 187

[12] A. Perelemov, Generalized Coherent States and Their Applications, SpringerVerlag Berlin 1986

[13] L.C. Biedenharn, and M.A. Lohe, Comm. Math. Phys. 146, 483 (1992)

F.H. Jackson, Messenger Math. 38, 57 (1909)

T.H. Koornwinder, Nederl. Acad. Wetensch. Proc. Ser. A92, 97 (1989)

D. I. Fivel, J. Phys. 24, 3575 (1991)

[14] M. Abramowitz and I.A. Stegun, Handbook of Mathematical Functions, Dover Publications, Inc., New York, 1972

[15] E. Celeghini, S. De Martino, S. De Siena, M. Rasetti and G. Vitiello, Mod. Phys. Lett. B7, 1321 (1993)

[16] J.R. Klauder and B. Skagerstam, Coherent States, World Scientific, Singapore 1985 
[17] V. Bargmann, P. Butera, L. Girardello and J.R. Klauder, Rep. on Math. Phys. 2 (1971) 221

[18] D. Mumford, Tata Lectures on Theta, III, Birkhäuser Boston, Inc., 1991

[19] R. Bellman, A brief introduction to theta functions, Holt, Rinehart and Winston, N.Y. 1961

[20] H.P. Yuen, Phys. Rev. 13 (1976) 2226

E. Celeghini, M. Rasetti, M. Tarlini and G. Vitiello, Mod. Phys. Lett. B3 (1989) 1213

[21] P. Carruthers and M. Nieto, Rev. Mod. Phys. 40 (1968) 411

[22] C. De Concini and G. Vitiello, Nucl. Phys. B116 (1976) 141

[23] R. Jackiw, J. Math. Phys. 9, (1968) 339

[24] E. Schrödinger, Naturwissenschaften 14 (1926) 664

[25] A.J. Dekker, Solid State Physics, Prentice-Hall, Englewood Cliffs 1957

[26] E. Celeghini, M. Rasetti and G. Vitiello, Annals of Phys.(N.Y.) 215 (1992) 156

[27] A. Iorio and G. Vitiello, Quantum groups and Von Neumann theorem, Mod. Phys. Lett. B, in press. 